\title{
Face transplantation for massive mandibular defects: considerations for allograft design and surgical planning
}

\author{
William Jackson Palmer ${ }^{1}$, Laurel Nelms ${ }^{2}$ \\ 'Boston University School of Medicine, Boston, MA 02118, USA. \\ 2University of California Riverside School of Medicine, Riverside, CA 92521, USA.
}

Correspondence to: Mr. William Jackson Palmer, Boston University School of Medicine, 72 E. Concord St., Boston, MA 02118, USA E-mail:wjpalmer@bu.edu

How to cite this article: Palmer WJ, Nelms L. Face transplantation for massive mandibular defects: considerations for allograft design and surgical planning. Plast Aesthet Res 2020;7:2. http://dx.doi.org/10.20517/2347-9264.2019.34

Received: 2 Oct 2019 First Decision: 30 Dec 2019 Revised: 7 Jan 2020 Accepted: 13 Jan 2020 Published: 20 Jan 2020

Science Editor: Ali-Farid Safi Copy Editor: Jing-Wen Zhang Production Editor: Tian Zhang

\begin{abstract}
Modern face transplant techniques have advanced to allow for the transfer of vascularized skeletal components in addition to overlying soft tissue. This represents significant opportunity for individuals with mandibular defects that are not amenable to traditional reconstruction. Care must be taken when planning and executing transplants with these complex grafts, as satisfactory functional and aesthetic outcomes rely on achieving proper spatial relationships between the mandible, skull base, and midface. Which donor skeletal elements are included in the allograft and how they are harvested are important considerations in this planning and are associated with controversy. To optimize outcomes in the reconstruction of single-jaw defects, some advocate for transplantation of only the affected jaw while others support bimaxillary transplantation. Clinical evidence in this debate is not conclusive at this time. In current practice, including donor dentoalveolar anatomy by utilizing a bilateral sagittal split osteotomy of the mandible is favored to optimize outcomes such as dental occlusion. It has been suggested that harvesting the mandible at the level of the condyle or even the temporal bone may also be possible and may improve temporomandibular joint-related outcomes. Despite encouraging preclinical evidence, these strategies remain controversial. After allograft design, successful mandibular reconstruction with face transplantation relies on surgical precision in the donor and recipient procedures. Computerized surgical planning, computer-aided design and manufacturing, and intraoperative navigation are technologies currently in use to mitigate operative complexity. Results in both cadaveric and clinical face transplantations suggest these technologies are reliable and beneficial, although some room for improvement remains.
\end{abstract}

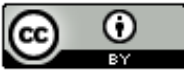

(C) The Author(s) 2020. Open Access This article is licensed under a Creative Commons Attribution 4.0 International License (https://creativecommons.org/licenses/by/4.0/), which permits unrestricted use, sharing, adaptation, distribution and reproduction in any medium or format, for any purpose, even commercially, as long as you give appropriate credit to the original author(s) and the source, provide a link to the Creative Commons license, and indicate if changes were made.

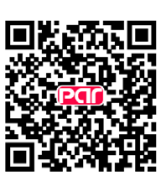


Keywords: Face transplantation, mandibular reconstruction, allograft design, bimaxillary transplantation, temporomandibular joint reconstruction, computerized surgical planning, computer-aided design and manufacturing, intraoperative navigation

\section{INTRODUCTION}

Face transplantation offers significant aesthetic and functional improvements for patients with devastating injuries that cannot be managed with conventional reconstruction ${ }^{[1-3]}$. Including the first procedure performed in 2005, 44 face transplantations have been reported in the scientific literature to date ${ }^{[2,4]}$. Successful transplantations have demonstrated the feasibility of this procedure and paved the way for technique refinement and increased operative complexity ${ }^{[3,4]}$. Surgeons are now able to include vascularized bone in addition to soft tissue in facial allografts ${ }^{[4,5]}$. The ability to incorporate varying amounts of donor facial skeleton allows the development of grafts that are customized to a patient's individual defect and reconstructive goals $s^{[2,5]}$. While many autologous reconstructive options for the mandible exist, they may fail to correct severe defects in some patients ${ }^{[6]}$. Modern face transplantation therefore represents a powerful tool for surgeons seeking to reconstruct massive facial defects involving the mandible.

Use of osteomyocutaneous allografts in this setting requires thorough planning and meticulous execution. To improve facial aesthetics and mandibular function, transplantation must not only replace missing or defective mandible; it is also necessary to restore proper spatial relationships between the mandible and other skeletal elements including the midface and skull base ${ }^{[4,7]}$. Establishing these relationships starts with thoughtful allograft planning based on an understanding of how the skeletal components included in the graft and the locations of osteotomies and osteosyntheses will influence functional outcomes. Precision in the subsequent steps of allograft harvest and inset is challenging and imperative. In many cases, recipients have endured injuries and reconstructive procedures that can disfigure anatomy and make graft alignment difficult ${ }^{[7,8]}$. Furthermore, when the recipient's defect warrants an allograft including both the midface and mandible, attachment of the graft is complicated by the paucity of recipient landmarks other than the skull base and the potential for misalignment in three dimensions ${ }^{[4,9]}$. Observed outcomes have reflected the procedural challenges, as transplants involving the maxilla and mandible have been associated with trismus, malocclusion, and impaired airway function, for example ${ }^{[4,7]}$. To avoid these functional complications and best restore this critical anatomy, transplants involving the mandible require detailed planning and precise execution ${ }^{[4]}$.

This review seeks to define some of the important considerations in the surgical planning of facial allografts involving the mandible as well as to highlight some of the emerging technologies available to optimize patient outcomes in these cases.

\section{DESIGNING THE MANDIBLE-CONTAINING ALLOGRAFT}

One of the earliest considerations in planning for facial transplantation to correct a mandibular defect is how much donor skeleton to include in the allograft. Given the goal of restoring function and appearance, it follows that, in the case of patients with defects involving both the maxilla and mandible, reconstruction with an allograft including both of these skeletal components is most appropriate ${ }^{[2,10]}$. Determining the extent of donor bone inclusion is more controversial in the case of patients with defects limited to only the mandible. In such circumstances, some advocate for transplantation of only the affected jaw while others support bimaxillary transplantation ${ }^{[4,11,12]}$.

In 2011, Gordon et al. ${ }^{[13,14]}$ introduced through cadaveric studies the concept of "hybrid occlusion", or the occlusal relationship achieved after allotransplanataion between the donor maxilla and recipient's 
native mandible. The concept of hybrid occlusion has since been extended to include cases where the native dentoalveolar structure is the maxilla, and the mandible is being transplanted ${ }^{[4,10]}$. Singlejaw transplantation resulting in hybrid occlusion has been utilized extensively in clinical cases of face transplantation $^{[11]}$. Proponents of this technique argue that poor occlusive outcomes may arise with singlejaw as well as with bimaxillary transplantation, but only the more conservative approach accounts for the risk of graft failure by preserving functional recipient anatomy ${ }^{[12,15]}$. Furthermore, these individuals suggest that occlusion can be improved with subsequent orthognathic and orthodontic interventions ${ }^{[12]}$. Those advocating for bimaxillary transplantation in cases of single-jaw defects cite the importance of adequate postoperative occlusion in restoring vital patient functions such as mastication and speech and suggest that preserving the donor occlusal relationship may optimize these outcomes ${ }^{[4,11]}$. Additionally, by obviating the need to find a donor jaw that properly fits the recipient's native jaw anatomy, bimaxillary transplantation may expand the possible donor pool ${ }^{[11]}$. No studies have directly compared single-jaw with bimaxillary transplantation, and the number of patients receiving these grafts is still relatively small; at this point, arguments for one strategy over the other based on functional outcomes remain experiential and speculative ${ }^{[4,11]}$.

In both mandible-only and bimaxillary transplantation, the next consideration is where to dissect the mandible. The earliest facial allotransplantation to utilize donor mandible included only the chin ${ }^{[16]}$. Subsequent procedures included donor mandible from angle to angle, which is now a common practice ${ }^{[16,17]}$. The bilateral sagittal split osteotomy of the mandible, a common procedure in traditional orthognathic surgery that involves splitting the mandible posterior to the alveolar process, has been widely incorporated into facial transplantation because it offers maximal donor-to-recipient bony contact ${ }^{[8,18]}$. Furthermore, this technique is favored over including only a portion of the mandibular tooth bed because incorporation of the entire donor alveolar structure is thought to better allow establishment of occlusion ${ }^{[10,18]}$. The exact path of bilateral sagittal split osteotomy can be customized for an individual recipient by using computer surgical planning techniques that are discussed below.

An exciting concept in the field currently is the prospect of transplanting the temporomandibular joint (TMJ) along with the mandible. Individuals with injuries warranting consideration of facial transplantation often have significant impairment of the $\mathrm{TMJ}^{[19]}$. Traumatic injuries themselves can cause scarring and other articular pathologies, and reconstructive efforts may similarly contribute to or exacerbate these conditions through scarring, reduction of jaw mobility, and subsequent muscle shortening ${ }^{[19]}$. Together, jaw injury and surgery may significantly impact a patient's quality of life by imposing difficulties opening the mouth, chewing, and speaking ${ }^{[19]}$. Donor mandibles void of articular anatomy, such as those prepared with the bilateral sagittal split osteotomy, do not alone address the potential for TMJ dysfunction in this population ${ }^{[16]}$. While it is possible to identify and treat TMJ pathology before facial transplantation, clinical evidence in the face transplant population suggests this strategy does not offer a definitive solution ${ }^{[4,19]}$. This fact is further complicated by the observation in at least one facial transplant candidate that TMJ impairments may not be apparent on preoperative imaging ${ }^{[19]}$. In cases where jaw dysfunction persists postoperatively, secondary revisions such as coronoidectomy and condylectomy are viable options that have been described in the literature with acceptable results ${ }^{[4,16,20]}$. In any case, transplantation with an allograft including the TMJ represents an alternative treatment option for these patients that may eventually offer comparable or superior functional outcomes and the possibility of fewer revision surgeries ${ }^{[16]}$.

The feasibility of TMJ transplantation and associated outcomes are still being elucidated. To date, there has been one case reported in the scientific literature of unilateral mandibular condyle inclusion in a facial allograft ${ }^{[6,16]}$. The patient in this case received a graft including donor mandible from right angle to left condyle to treat a large mandibular defect and known left TMJ ankylosis secondary to radiation therapy ${ }^{[6,16]}$. The transplantation was successful, and, although the patient demonstrated only $10 \mathrm{~mm}$ of mandibular 
excursion postoperatively, this was attributed to prior right TMJ injury ${ }^{[6,16]}$. No facial transplantation reported to date has included both mandibular condyles, and, accordingly, the feasibility and practicality of this procedure remain unclear ${ }^{[4,16]}$. For adequate postoperative jaw function in the case of allografts including both donor mandibular condyles, the transplanted segment must align with the recipient's glenoid fossae in a way that enables functional articulation ${ }^{[16]}$. It follows that this may make donor selection burdensome, as mandible morphology including intercondylar distance is known to vary significantly between individuals ${ }^{[4,16]}$. However, preclinical evidence has been encouraging. Khavanin et al. ${ }^{[16]}$ utilized the computed tomography (CT) scans of 100 adults to evaluate the viability of bilateral condylar transplant and concluded that the procedure would be anatomically feasible and clinically practical in most cases given adequate average interglenoid widths and the fact that the glenoid fossa itself is wider than the mandibular condyle $^{[4]}$. It should be noted that this study excluded individuals with mandibular trauma or defects ${ }^{[16]}$. Candidates for mandibular condyle transplantation may have anatomical changes in the glenoid region that impact the feasibility of accepting a donor condyle. More targeted research is warranted. Bilateral condyle transplantation has also been challenged on the grounds that violating the TMJ's supportive connective tissue may result in complications such as ankylosis or joint instability ${ }^{[4]}$. As a potential solution to these concerns, it has been suggested that the entire TMJ including donor temporal bone could be transplanted, although this would increase procedural complexity and operative time ${ }^{[16]}$. Additionally, the vascular anastomoses necessary to support this anatomy have not been demonstrated, and until outcomes of these procedures are reported, suggesting the superiority of one method over the other remains speculation ${ }^{[4,16]}$. With further characterization, transplanting the TMJ along with the mandible may become a valuable reconstructive modality for individuals with severe mandibular defects and impairments ${ }^{[16]}$.

\section{OPTIMIZING SURGICAL PRECISION}

Once the skeletal components of the allograft and the general osteotomy locations have been determined, two operative procedures must be completed: the donor harvest and the recipient inset ${ }^{[8]}$. Both of these procedures are technically challenging and time-intensive ${ }^{[8,21]}$. The donor harvest demands a thorough understanding of the recipient's defect and an appropriate surgical plan; otherwise, the surgical team would be faced with the time-consuming and error-prone challenge of adjusting the allograft to fit the recipient during transplantation ${ }^{[7,21]}$. The recipient procedure, including preparation and allograft attachment, requires similar precision to yield proper spatial relationships between regional anatomy, a crucial factor in the restoration of aesthetics and functional parameters such as dental occlusion ${ }^{[5,7,8,22-24]}$. Achieving proper alignment between the allograft and the recipient's native anatomy may be particularly challenging in face transplantation, where devastating injuries and previous reconstructive efforts can mar regional anatomy ${ }^{[7,8]}$. To address these challenges, surgical teams have begun incorporating computer-based technology, including computerized surgical planning (CSP), computer-aided design and manufacturing $(\mathrm{CAD} / \mathrm{CAM})$, and intraoperative navigation ${ }^{[4,8,21]}$.

Prior to their use in facial transplantation, these computer-aided techniques demonstrated promise in other craniomaxillofacial applications ${ }^{[8]}$. With this technology, preoperative CT images can be rendered in three dimensions in a virtual workspace, allowing the user to develop a surgical plan based on virtual manipulation and measurement of the patient's anatomy ${ }^{[22]}$. This technology obviates the need to design a three-dimensional plan off of two-dimensional images ${ }^{[2]}$. Tangible stereolithographic models may also be produced from these virtual representations to further aid in operative planning ${ }^{[22]}$. In the case of fibular free flaps for midface or mandibular reconstruction, for instance, the osteotomies can be designed virtually, corresponding measurements can be used to produce stereolithographic models of these skeletal elements (a technique termed CAD/CAM), and reconstructive hardware can be pre-formed to these patient-specific models prior to surgery ${ }^{[24]}$. The virtual measurements can also be used to design patientspecific cutting guides that facilitate accurate osteotomies in the operating room ${ }^{[22]}$. Advancing virtual reality technology and the incorporation of haptic feedback devices into virtual workstations promise to 
make the surgical planning process even more immersive, realistic, and accurate ${ }^{[25]}$. Virtual rendering of patient images has also offered intraoperative and postoperative utility. Intraoperative navigation, which uses a portable localizer and patient CT scans to show the surgeon the real-time position of an instrument, has demonstrated the ability to accurately locate anatomy and surgical tools within a small margin of error $^{[22]}$. This strategy may be particularly useful in cases of structures that are challenging to visualize intraoperatively such as parts of the mandible and the base of the skull ${ }^{[22]}$. Augmented reality, which allows for the projection of computer-generated images onto the surgical field in real time, is another promising computer-based technology that has been applied in various areas of maxillofacial surgery to improve intraoperative navigation in challenging anatomical scenarios ${ }^{[25,26]}$. Postoperatively, three-dimensional rendering of CT scans can be compared via virtual superimposition on surgical plans to confirm results and evaluate fidelity to the preoperative plan $^{[21,22]}$. These computer-aided tools have reduced surgical time and improved precision in multiple craniomaxillofacial applications, making them an attractive technology for face transplantation ${ }^{[8,24]}$.

Jacobs et al. ${ }^{[21]}$ proposed and validated through cadaveric transplantation a planning protocol using CSP and CAD/CAM technologies that reflects their use in the face transplantation field at large and depicts their utility. While this team focused on allografts without mandible, their principles have implications for mandibular reconstruction as accurate handling of the maxilla is necessary for the establishment of a functional maxillomandibular relationship ${ }^{[21]}$. Furthermore, several teams have used similar protocols with allografts including varying amounts of donor mandible ${ }^{[8,9,27]}$. First, using preoperative CT images rendered in three dimensions, the recipient's defect is defined ${ }^{[21]}$. In the virtual workspace, the donor rendering can then be superimposed on the recipient in order to plan appropriate osteotomies ${ }^{[21]}$. Jacobs et al. ${ }^{[21]}$ advocate for first virtually establishing optimal donor-to-recipient bony relationships including occlusion and then using the resulting model to design osteotomy paths that will yield these exact relationships. Other teams have since suggested that the osteotomies be defined in a way that preserves functional soft tissue ${ }^{[27]}$. In either case, once the osteotomy is depicted virtually, custom cutting guides based on these models can be manufactured to enable efficient and precise osteotomies designed specifically to establish these predetermined anatomical relationships ${ }^{[21,27]}$. In the case of single-jaw transplantation, a custom dental splint can also be prepared based on the virtual model to further aid in establishment of proper occlusion intraoperatively ${ }^{[21]}$. In cases of bimaxillary transplantation where the landmark of a native recipient jaw is absent, the donor jaws can be placed in intermaxillary fixation preoperatively for a similar effect ${ }^{[4,8,9]}$. Finally, the skeletal aspects of the transplantation procedure can be conducted in the virtual workspace to assess outcomes and refine the plan as necessary ${ }^{[21]}$.

Using the postoperative overlay analysis described previously, Jacobs et al.$^{[21]}$ found that the surgical result of their midface transplantation differed from the virtual plan by less than $5 \mathrm{~mm}$. In a series of seven cadaveric transplantations including a portion of the mandible, Sosin et al. ${ }^{[27]}$ utilized CSP and CAD/CAM and also demonstrated close adherence of the postoperative results to preoperative plans. This team also found that grafts prepared and attached based on these virtual plans did not require time-consuming ad hoc intraoperative adjustments ${ }^{[27]}$. This corroborates the idea that virtual surgical plans can be reproduced reliably in the operating room with a time-saving benefit ${ }^{[27]}$.

Intraoperative applications of computer-based technologies have also been validated through cadaveric face transplant models. As mentioned above, computer-based strategies that enable precise alignment of skeletal components may be particularly useful in face transplants that include both the midface and mandible, as recipients in these cases may lack obvious landmarks to guide graft attachment ${ }^{[9]}$. Brown et al. ${ }^{[8]}$ preformed 10 bimaxillary face transplantations based on CSP principles. In addition to using CSP to plan the osteotomies and manufacture cutting guides, the team utilized a stereolithographic model of the recipient as well as intraoperative navigation during osteotomies and graft inset ${ }^{[8]}$. The stereolithographic model 
was constructed from a representation of the recipient after virtual osteotomy; in other words, the model represented the recipient's facial skeleton prior to graft inset ${ }^{[8]}$. During the donor harvest, the proposed allograft was aligned to this stereolithographic model before severing the graft's supporting vasculature ${ }^{[8]}$. This technique allowed the surgical team to optimize the fit and adapt surgical hardware in advance of ischemia time ${ }^{[8]}$. The team demonstrated no significant difference in cephalometric variables including occlusal plane angles between the virtual surgical plans and the postoperative results ${ }^{[8]}$. Postoperative occlusion was also successfully achieved ${ }^{[8]}$. Dorafshar et al ${ }^{[9]}$ demonstrated similarly encouraging results in their study of five bimaxillary cadaver transplants conducted with donor osteotomies under intraoperative navigation and recipient osteotomies facilitated by custom-manufactured cutting guides. This team found that the postoperative results closely mirrored the computer-designed surgical plan in five of the six axes of movement with significant differences only appearing in lateral translation ${ }^{[9]}$.

Fidelity to the virtual surgical plan has also been demonstrated in several clinical face transplantations. Sosin et al. ${ }^{[28]}$ successfully performed a facial transplantation including the genial segment of the mandible with CSP and CAD/CAM and noted postoperative results in close agreement with their virtual plan. Dorafshar et al. ${ }^{[18]}$ utilized cutting guides, intraoperative navigation, and donor alignment to a stereolithographic model of the recipient in their double-jaw transplantation with high fidelity to the virtual plan and postoperative maintenance of occlusion. Dorafshar et al ${ }^{[9]}$ also followed their five cadaveric transplants with a clinical transplant employing the same protocol and noted similar postoperative results between the two groups. More recently, Ramly et al. ${ }^{[4]}$ reported two bimaxillary transplants facilitated by CSP, CAD/CAM, and intraoperative navigation that both resulted in adherence to the virtual surgical plan and class I occlusion following transplantation. Together, these results support the feasibility of conducting face transplantation with computer-aided strategies and achieving reliable postoperative results. As these surgical plans are tailored to the reconstructive goals of each recipient-donor pair, it follows that technology enabling the accurate reproduction of these plans should optimize patient outcomes ${ }^{[7]}$.

While evidence to date supports the role of CSP and other computer-based strategies in maximizing operative efficiency and optimizing postoperative face transplantation results, there remains room for improvement ${ }^{[5]}$. The evidence provided by Dorafshar et al. ${ }^{[9]}$ serves as a reminder that a degree of infidelity between the virtual plan and the postoperative results exists. These authors suggest that fidelity to the virtual plan may improve as teams become more comfortable with CSP strategies but that the surgeon will likely still be required to make decisions based on intraoperative observations, particularly in cases of challenging anatomy ${ }^{[9]}$. It is also important to understand that while encouraging, fidelity to a virtual plan does not necessarily result in enduring spatial relationships. Current CSP technology is not equipped to accurately account for the influence of recipient musculature on postoperative allograft positioning ${ }^{[4,5,11]}$. In the two clinical bimaxillary transplants reported by Ramly et al. ${ }^{[4]}$, both patients developed malocclusion during their recoveries despite the class I occlusion observed after transplantation. One of these patients also subsequently required mandibular coronoidectomy to improve mandibular mobility ${ }^{[4]}$. Improved CSP software that can predict dynamic bone-to-bone relationships in the context of postoperative muscle recovery may help to improve long-term occlusive and TMJ outcomes ${ }^{[4]}$.

\section{CONCLUSION}

Face transplantation techniques have evolved to allow for the inclusion of vascularized bone in the donor allograft. For individuals with large, complex facial defects involving the mandible, these grafts represent a valid and important option for reconstruction when other strategies fail. Thoughtful allograft design, thorough surgical planning, and precise execution of these procedures are necessary to ensure proper postoperative relationships between the mandible and the maxilla as well as the mandible and the skull base. By establishing proper relationships between these skeletal elements, surgeons can restore the aesthetics and functionalities lost with massive mandibular injury. Transplantation of the TMJ and 
computer-aided planning, manufacturing, and navigation are emerging technologies that, with deeper understanding and continued refinement, appear to promise these patients improved outcomes.

\section{DECLARATIONS}

\section{Authors' contributions}

Made substantial contributions to conception and design, analysis, interpretation, and preparation of the review and manuscript: Palmer WJ

Assisted with manuscript preparation, as well as provided administrative, technical, and material support: Nelms L

\section{Availability of data and materials}

Not applicable.

\section{Financial support and sponsorship}

None.

\section{Conflicts of interest}

All authors declared that there are no conflicts of interest.

\section{Ethical approval and consent to participate}

Not applicable.

\section{Consent for publication}

Not applicable.

\section{Copyright}

(c) The Author(s) 2020.

\section{REFERENCES}

1. Fischer S, Lee TC, Krezdorn N, Alhefzi M, Kueckelhaus M, et al. First lower two-thirds osteomyocutaneous facial allograft perfused by a unilateral facial artery: outcomes and vascularization at 1 year after transplantation. Plast Reconstr Surg 2017;139:1175e-83e.

2. Pomahac B, Nowinski D, Diaz-Siso JR, Bueno EM, Talbot SG, et al. Face transplantation. Curr Probl Surg 2011;48:293-357.

3. Infante-Cossio P, Barrera-Pulido F, Gomez-Cia T, Sicilia-Castro D, Garcia-Perla-Garcia A, et al. Current role in facial allograft transplantation: what have we learned? Plast Aesthet Res 2016;3:211-8.

4. Ramly EP, Kantar RS, Diaz-Siso JR, Alfonso AR, Shetye PR, et al. Outcomes after tooth-bearing maxillomandibular facial transplantation: insights and lessons learned. J Oral Maxillofac Surg 2019;77:2085-103.

5. Rifkin WJ, David JA, Plana NM, Kantar RS, Diaz-Siso JR, et al. Achievements and challenges in facial transplantation. Ann Surg 2018;268:260-70.

6. Cavadas PC, Ibáñez J, Thione A. Surgical aspects of a lower face, mandible, and tongue allotransplantation. J Reconstr Microsurg 2012;28:43-7.

7. Plana NM, Malta Barbosa J, Diaz-Siso JR, Brecht LE, Rodriguez ED. Dental considerations and the role of prosthodontics and maxillofacial prosthetics in facial transplantation. J Am Dent Assoc 2018;149:90-9.

8. Brown EN, Dorafshar AH, Bojovic B, Christy MR, Borsuk DE, et al. Total face, double jaw, and tongue transplant simulation: a cadaveric study using computer-assisted techniques. Plast Reconstr Surg 2012;130:815-23.

9. Dorafshar AH, Brazio PS, Mundinger GS, Mohan R, Brown EN, et al. Found in space: computer-assisted orthognathic alignment of a total face allograft in six degrees of freedom. J Oral Maxillofac Surg 2014;72:1788-800.

10. Mohan R, Borsuk DE, Dorafshar AH, Wang HD, Bojovic B, et al. Aesthetic and functional facial transplantation: a classification system and treatment algorithm. Plast Reconstr Surg 2014;133:386-97.

11. Khalifian S, Brazio PS, Mohan R, Shaffer C, Brandacher G, et al. Facial transplantation: the first 9 years. Lancet 2014;384:2153-63.

12. Wall A, Bueno E, Pomahac B, Treister N. Intraoral features and considerations in face transplantation. Oral Dis 2016;22:93-103.

13. Gordon CR, Susarla SM, Peacock ZS, Kaban LB, Yaremchuk MJ. Le Fort-based maxillofacial transplantation: current state of the art and a refined technique using orthognathic applications. J Craniofac Surg 2012;23:81-7.

14. Gordon CR, Susarla SM, Peacock ZS, Cetrulo CL, Zins JE, et al. Osteocutaneous maxillofacial allotransplantation: lessons learned from a novel cadaver study applying orthognathic principles and practice. Plast Reconstr Surg 2011;128:465e-79e. 
15. Pomahac B, Bueno EM, Sisk GC, Pribaz JJ. Current principles of facial allotransplantation: the Brigham and Women's Hospital Experience. Plast Reconstr Surg 2013;131:1069-76.

16. Khavanin N, Davidson EH, Lee DY, Byrne P, Dorafshar AH. Anatomic considerations for temporomandibular joint vascularized composite allotransplantation. J Craniofac Surg 2018;29:871-7.

17. Lantieri L, Hivelin M, Audard V, Benjoar MD, Meningaud JP, et al. Feasibility, reproducibility, risks and benefits of face transplantation: a prospective study of outcomes. Am J Transplant 2011;11:367-78.

18. Dorafshar A, Bojovic B, Christy M, Borsuk D, Iliff N, et al. Total face, double jaw, and tongue transplantation: an evolutionary concept. Plast Reconstr Surg 2013;131:241-51.

19. Krezdorn N, Alhefzi M, Perry B, Aycart MA, Tasigiorgos S, et al. Trismus in face transplantation following ballistic trauma. J Craniofac Surg 2018;29:843-7.

20. Aycart MA, Alhefzi M, Kueckelhaus M, Krezdorn N, Bueno EM, et al. A retrospective analysis of secondary revisions after face transplantation: assessment of outcomes, safety, and feasibility. Plast Reconstr Surg 2016;138:690e-701e.

21. Jacobs JMS, Dec W, Levine JP, McCarthy JG, Weimer K, et al. Best face forward: virtual modeling and custom device fabrication to optimize craniofacial vascularized composite allotransplantation. Plast Reconstr Surg 2013;131:64-70.

22. Bell RB. Computer planning and intraoperative navigation in cranio-maxillofacial surgery. Oral Maxillofac Surg Clin North Am 2010;22:135-56

23. Caterson EJ, Diaz-Siso JR, Shetye P, Junker JPE, Bueno EM, et al. Craniofacial principles in face transplantation. J Craniofac Surg 2012;23:1234-8.

24. Rudman K, Hoekzema C, Rhee J. Computer-assisted innovations in craniofacial surgery. Facial Plast Surg 2011;27:358-65.

25. Kim Y, Kim H, Kim YO. Virtual reality and augmented reality in plastic surgery: a review. Arch Plast Surg 2017;44:179-87.

26. Wong K, Yee HM, Xavier BA, Grillone GA. Applications of augmented reality in otolaryngology: a systematic review. Otolaryngol Head Neck Surg 2018;159:956-67.

27. Sosin M, Ceradini DJ, Hazen A, Levine JP, Staffenberg DA, et al. Total face, eyelids, ears, scalp, and skeletal subunit transplant cadaver simulation: the culmination of aesthetic, craniofacial, and microsurgery principles. Plast Reconstr Surg 2016;137:1569-81.

28. Sosin M, Ceradini DJ, Levine JP, Hazen A, Staffenberg DA, et al. Total face, eyelids, ears, scalp, and skeletal subunit transplant: a reconstructive solution for the full face and total scalp burn. Plast Reconstr Surg 2016;138:205-19. 